\title{
Caffeine promotes premature chromosome condensation formation and in vitro development in porcine reconstructed embryos via a high level of maturation promoting factor activity during nuclear transfer
}

Manabu Kawahara, Takuya Wakai, Ken-Ichi Yamanaka, Jin Kobayashi ${ }^{1}$, Satoshi Sugimura, Takashi Shimizu, Hiromichi Matsumoto, Jin-Hoi Kim², Hiroshi Sasada and Eimei Sato

Laboratory of Animal Reproduction, Graduate School of Agricultural Science, Tohoku University, Sendai 981-8555, Japan, ${ }^{1}$ Research Farm, Miyagi Agricultural College, Sendai, Japan and ${ }^{2}$ Division of Applied Life Science, GyeongSang National University, Chinju, GyeongNam, Korea

Correspondence should be addressed to M Kawahara, Department of Bioscience, Tokyo University of Agriculture, 1-1-1 Sakuragaoka, Setagaya-ku, Tokyo 156-8502, Japan; Email: mana1976@nodai.ac.jp

\begin{abstract}
When the nucleus in G0/G1 phase is transferred to an enucleated oocyte by nuclear transfer (NT), its nuclear envelope is broken, followed by condensation of chromosome structure by maturation promoting factor (MPF). This morphological remodeling of the transferred interphase nucleus seems to be essential for subsequent development of NT embryos. In this study, we treated porcine NT embryos with caffeine, which has been reported to increase MPF activity, to keep their MPF level high during NT. When $2.5 \mathrm{mM}$ caffeine was added to the handling medium, the proportion of NT embryos showing condensed chromosome increased significantly $(P<0.05)$. In NT embryos treated with caffeine, the activity of p34 ${ }^{\text {cdc2 }}$ kinase was significantly $(P<0.05)$ higher than in those without caffeine at $3 \mathrm{~h}$ post-injection. In addition, the rate of development to the blastocyst stage after activation was significantly $(P<0.05)$ higher in NT embryos treated with caffeine. These results indicate that caffeine treatment can increase not only the rate of chromosome condensation but also the developmental rate to the blastocyst stage of porcine NT embryos. This action is most likely due to the support/increase of MPF activity throughout the process of NT.
\end{abstract}

Reproduction (2005) 130 351-357

\section{Introduction}

Pig cloning may facilitate many biomedical applications, such as xenotransplantation, bioreactor and a model animal in human disease (Prather et al. 2003). Although there has been recent success of pig cloning, the efficiency is still low, as it is in other animals (Boquest et al. 2002, De Sousa et al. 2002, Yin et al. 2002). One of the reasons for the very low success rate is the insufficient reprogramming of the transferred nucleus in the current nuclear transfer (NT) system (Kang et al. 2001). At present, the mechanism(s) of reprogramming for the differentiated nucleus is still unknown.

When the donor nuclei in G0/G1 of the cell cycle are transferred into recipient cytoplasts with a high level of maturation promoting factor (MPF) before activation, they are transformed to nuclear envelope breakdown (NEBD) and then premature chromosome condensation (PCC) (Ikeda \& Takahashi 2001). In the mouse and bovine, this
PCC of the transferred nucleus was related to the success of cloning (Shin et al. 2002, Wakayama et al. 1998). Therefore, MPF activity in recipient cytoplasts and NT embryos seems to be an important factor for development of NT embryos. It has been reported that MPF activity decreases after prolonged culture or artificial activation of the oocyte (Kikuchi et al. 1995, Machaty \& Prather 1998). When oocytes with low MPF activity are fertilized with sperm, they show abnormal pronuclear formation and embryo development, indicating that MPF activity is thought as an indicator to evaluate the quality of the oocyte (Kikuchi et al. 1995). However, MPF activity in recipient cytoplasts and NT embryos has remained poorly understood, although the maturational cultivation period of the oocytes used as recipient cytoplasts can affect the developmental ability of NT embryos (Cheong et al. 2000, Ikeda \& Takahashi 2001, Miyoshi et al. 2002). Furthermore, the relationship between morphological remodeling such as NEBD or PCC 
and the development after activation has been poorly understood (Uhm et al. 2000). Enucleation, microinjection and manipulation time are also likely to have detrimental effects upon MPF activity of recipient cytoplasts and NT embryos. Thus, it is interesting how MPF activity changes during NT, and whether its change would affect the development of NT embryos in pigs.

One of the mechanisms that regulate MPF activity is a modification of the phosphorylation status of p34 $4^{\text {cdc2 }}$ kinase by Myt1/Wee1 and cdc25. The addition of caffeine into the culture medium suppresses Myt1/Wee1 activity, resulting in an inhibition of a shift from active MPF to preMPF, the inactive form, and an increase of MPF activity (Kikuchi et al. 2002). Kikuchi et al. (2000) showed the inhibition of the decrease of MPF activity in pig oocytes by the supplement of caffeine. Therefore, there is a possibility that a high level of MPF caused by caffeine in recipient cytoplasts and/or NT embryos can promote PCC of NT embryos in pigs. In the present study, we examined the effect of caffeine on morphological remodeling, such as NEBD or PCC, and the development in porcine NT embryos.

\section{Materials and Methods}

\section{Preparation of oocytes matured in vitro}

Pig cumulus-oocytes complexes (COCs) were isolated as reported by Kawahara et al. (2002). In brief, COCs were aspirated from nonatretic follicles $3-5 \mathrm{~mm}$ in diameter of ovaries, which were obtained from prepubertal gilts at a local slaughterhouse. After washing in PB1, 10 COCs each were cultured in droplets of BSA-free NCSU-23 medium for $44 \mathrm{~h}$ at $38.5^{\circ} \mathrm{C}$ in a humidified atmosphere of $5 \% \mathrm{CO}_{2}$ in air. During the first $22 \mathrm{~h}$ of maturation, the medium contained $10 \mathrm{lU} / \mathrm{ml}$ PMSG (Serotropin, Teikokuzouki, Tokyo, Japan), $10 \mathrm{lU} / \mathrm{ml}$ hCG (Puberogen, Sankyo, Tokyo, Japan), $0.1 \mathrm{mg} / \mathrm{ml}$ cysteine (Sigma Chemical Co., St Louis, MO, USA), $5 \mathrm{mg} / \mathrm{ml}$ insulin (GIBCO BRL, Life Technologies INC., Grand Island, NY, USA), $25 \mu \mathrm{M} \beta$-mercaptoethanol (Sigma) and 10\% (v/v) porcine follicular fluid. Culture for the subsequent $22 \mathrm{~h}$ was carried out in the same medium without hormonal supplementation. After the maturational cultivation, expanded cumulus cells were removed by vortexing in PB1 containing $1 \mathrm{mg} / \mathrm{ml}$ hyaluronidase (Sigma), and the oocytes with the first polar body were selected as matured oocytes under a stereomicroscope. The mature oocytes were placed in PB1 until they were used.

\section{Donor cells}

Miniature pig fetuses (Gottingen, Chugai Co., Suwa, Japan) were collected from a pregnant gilt at day 56 of pregnancy. The intact uterus was excised from the gilt and was immediately transported to the laboratory for recovery of fetuses. Each fetus was decapitated and eviscerated. The remaining tissue was washed in Dulbecco's PBS (Nissui, Tokyo, Japan) and then was digested by $0.1 \%$
trypsin-EDTA for $45 \mathrm{~min}$ at $38.5^{\circ} \mathrm{C}$. Following the digestion, the cells were cultured in Dulbecco's modified Eagle medium (DMEM, Sigma) supplemented with 10\% fetal bovine serum (FBS, Sigma). The culture medium was changed every 2 days until the cells reached confluence. The cells were then harvested by trypsinization with $0.1 \%$ trypsin-EDTA for $5 \mathrm{~min}$ at $38.5^{\circ} \mathrm{C}$, frozen in cell conservation medium (Cell banker, Nihon Zenyaku Co., Tokyo, Japan) and stored in liquid nitrogen (passage 0). For experiments, the cells from a single fetus were thawed and cultured in DMEM plus 10\% FBS and used at passages 4-9. Before use as donor nuclei, the cells were cultured for 1 week after confluence.

\section{Enucleation and microinjection}

Oocytes stripped of cumulus cells were stained by $5 \mu \mathrm{g} / \mathrm{ml}$ Hoechst 33342 (Sigma) at $38.5^{\circ} \mathrm{C}$ for 5 to $10 \mathrm{~min}$ and manually enucleated in PB1 (Quinn et al. 1982) containing $7.5 \mu \mathrm{g} / \mathrm{ml}$ Cytochalasin B (Sigma). Enucleation was performed by aspirating the first polar body and adjacent cytoplasm using a beveled pipette driven by a piezo-actuated unit (Prime Tech, Ibaraki, Japan), and confirmed by visualizing the cytoplasm under UV light. Enucleated oocytes were washed three times and transferred to $50 \mu$ l droplets of PB1 for microinjection of donor nuclei. After enucleation, donor cells were resuspended in PB1 after trypsinization. They were transferred to a $50 \mu$ l droplet of PB1 for microinjection, and their cell broken by gentle aspiration of the entire cell into and out of the beveled pipette. After the microinjection of the donor nuclei, NT embryos were cultured at $38.5^{\circ} \mathrm{C}$ in $100 \mu$ of NCSU23 containing $4 \mathrm{mg} / \mathrm{ml}$ BSA for approximately $3 \mathrm{~h}$ before activation.

\section{Activation and culture of NT embryos}

Three hours after microinjection, NT embryos were activated by ionomycin (Sigma), electrical stimulation and cycloheximide (Sigma). To activate NT embryos with ionomycin, they were treated with $15 \mu \mathrm{M}$ ionomycin in NCSU23 for $20 \mathrm{~min}$ at $38.5^{\circ} \mathrm{C}$ in $5 \% \mathrm{CO}_{2}$ in humidified air, and then washed five times with NCSU23. After treatment, electrical stimulation was applied with an LF 101 (TR Tech, Tokyo, Japan) using a chamber with two parallel platinum wire electrodes spaced $1 \mathrm{~mm}$ apart overlain with activation medium $(300 \mathrm{mM}$ mannitol supplemented with $0.01 \%$ polyvinyl alcohol, $0.05 \mathrm{mM} \mathrm{CaCl} 2 \cdot 2 \mathrm{H}_{2} \mathrm{O}$, and $0.1 \mathrm{mM} \mathrm{MgSO} \cdot \cdot 7 \mathrm{H}_{2} \mathrm{O}$, all from Wako Pure Chemical Industries Ltd, Osaka, Japan). Before electrical stimulation, the oocytes were equilibrated in activation medium for $5 \mathrm{~min}$. Double DC pulses of $1.2 \mathrm{kV} / \mathrm{cm}$ for $60 \mu \mathrm{sec}$ separated by $5 \mathrm{sec}$ were used for electrical stimulation. After the stimuli, the oocytes were transferred to NCSU23 and cultured at $38.5^{\circ} \mathrm{C}$ in an atmosphere of $5 \% \mathrm{CO}_{2}$ in air. Lastly, NT embryos were cultured in NCSU23 containing $5 \mu \mathrm{g} / \mathrm{ml}$ cycloheximide and $2.5 \mu \mathrm{g} / \mathrm{ml}$ cytochalasin D for 
$5 \mathrm{~h}$ at $38.5^{\circ} \mathrm{C}$ in $5 \% \mathrm{CO}_{2}$ in humidified air, and then washed 5 times with cycloheximide-free NCSU23.

Activated NT embryos were transferred into NCSU23 and cultured for 7 days. NT embryos were examined for cleavage and blastocyst formation at 2 and 7 days of culture, respectively. At the end of the culture period, all blastocysts were counted for nuclei after $5 \mu \mathrm{g} / \mathrm{ml}$ Hoechst staining. The numbers of nuclei were counted under ultraviolet light.

\section{In vitro p34 ${ }^{c d c 2}$ kinase assay}

We performed the p34 ${ }^{\text {cdc2 }}$ kinase assay using a Mesacup cdc2 kinase assay kit (code no. 5234; MBL, Nagoya, Japan) as described by Shimada et al. (2001). Briefly, $7 \mu l$ of five oocytes or NT embryos extract was mixed with $17.5 \mu \mathrm{l}$ of kinase assay buffer with $10 \%(\mathrm{v} / \mathrm{v}) \mathrm{MV}$ peptide solution and $0.1 \mathrm{mM}$ ATP (Sigma). The mixture was incubated for $30 \mathrm{~min}$ at $30^{\circ} \mathrm{C}$, and the reaction was terminated by phosphorylation stop reagent. Phosphorylation was detected using an ELISA analysis. Values were expressed as the fold strength of p34 ${ }^{\text {cdc2 }}$ kinase in oocytes just after collection from follicles, defined as 1 . Assays for the same experiments were performed all at once.

\section{Experimental studies}

In order to examine the time course of PCC appearance and the effect of caffeine on the appearance rate of PCC, the states of transferred nuclei were examined after the cultivation for $0,1.5,3$ and $4.5 \mathrm{~h}$ after microinjection with or without caffeine in experiment 1 . After the culture for each period, degenerated NT embryos with collapsed membranes were removed. The NT embryos were mounted, fixed for $48 \mathrm{~h}$ in $25 \%(\mathrm{v} / \mathrm{v})$ acetic acid in ethanol at room temperature, stained with $1 \%(\mathrm{w} / \mathrm{v})$ orcein in $45 \%$ $(\mathrm{v} / \mathrm{v})$ acetic acid, and the state of the transferred nucleus in each embryo was examined under a phase-contrast microscope. We categorized the state of the transferred nucleus based on Fig. 1. In brief, oocytes that had nuclei in $\mathrm{E}$ or $\mathrm{F}$ in Fig. 1 were categorized as NEBD, and those that had nuclei in $\mathrm{G}$ or $\mathrm{H}$ were categorized as PCC.
In experiment 2, the effect of caffeine on p34 ${ }^{\text {cdc2 }}$ kinase activity in porcine oocytes matured in vitro was examined. In addition, the effect of caffeine on p34 $4^{\text {cdc2 }}$ kinase activity of enucleated oocytes and oocytes injected with donor nuclei were examined. First, we assayed p34 $4^{\text {cdc2 }}$ kinase activity in the matured oocytes cultured for $44 \mathrm{~h}$. Secondly, after the maturational culture for $44 \mathrm{~h}$, the oocytes were cultured in PB1 with or without $2.5 \mathrm{mM}$ caffeine for $8 \mathrm{~h}$ further, and their p34 ${ }^{\text {cdc2 }}$ kinase activities were assayed. Matured oocytes were placed in PB1 and NCSU23 with or without $2.5 \mathrm{mM}$ caffeine from enucleation to the time $3 \mathrm{~h}$ after microinjection. Caffeine was also added in the manipulation medium. We assayed p34 $4^{\text {cdc2 }}$ kinase activity of enucleated and injected oocytes.

Experiment 3 was undertaken to evaluate the effect of caffeine on development of NT embryos. As described in experiment 2, NT embryos were produced in medium with or without $2.5 \mathrm{mM}$ caffeine until they were activated at 0 and $3 \mathrm{~h}$ post-injection, and examined for cleavage and blastocyst formation at 2 and 7 days of culture, respectively. As the control to NT embryos, in vitro maturation, fertilization and culture (IVMFC) of porcine oocytes as described by Miyoshi et al. (1999) was performed to produce blastocysts. The IVF medium also contained $2.0 \mathrm{mM}$ caffeine to induce capacitation and spontaneous acrosome reactions of boar spermatozoa (Funahashi et al. 2000).

Experiment 4 was performed to examine whether the addition of caffeine affected the formation of abnormal pseudopronucleus in the NT embryos without activation. In the same way as experiment 1 , the states of transferred nuclei were examined after the cultivation for 3 and $4.5 \mathrm{~h}$ after microinjection with or without caffeine by orceinstaining.

\section{Statistical analysis}

All data were replicated three times. The proportion of NT embryos showing PCC with or without caffeine treatment was analyzed for statistically significant differences with $t$-test, the other data analyzed with ANOVA followed by Fisher's protected least significant differences $(P<0.05)$ (StatView, SAS Institute Inc., Cary, NC, USA).

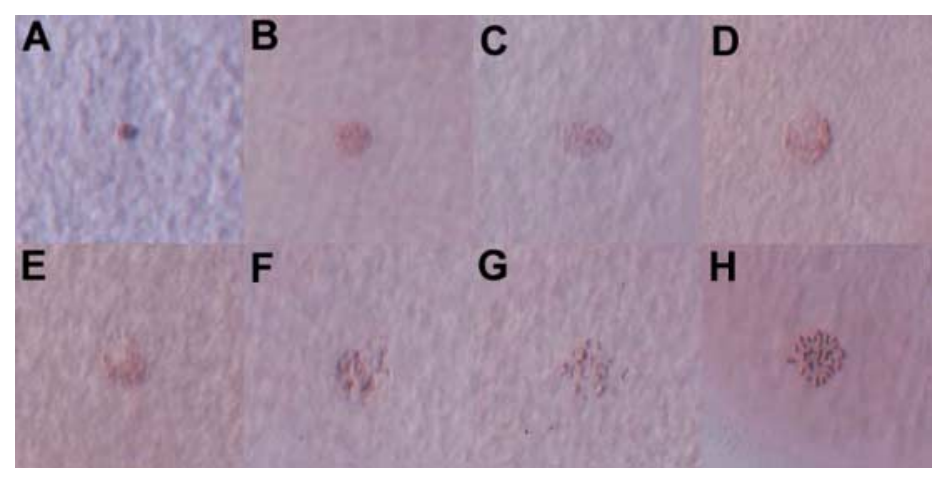

Figure 1 States of nuclei transferred after microinjection to enucleated oocytes. (A-D) Immediate state post-injection, (E and F) Nuclear envelope breakdown at $1.5 \mathrm{~h}$ post-injection. $(\mathrm{G}$ and $\mathrm{H}$ ) Prematurely condensed nuclei at $3 \mathrm{~h}$ post-injection. 


\section{Results}

Time course of PCC appearance and effect of caffeine on proportion of NT embryos showing PCC from 0 to 4.5 $\mathrm{h}$ after microinjection

The state of the transferred nucleus in each NT embryo was examined after the cultivation with or without caffeine immediately, 1.5, 3 and $4.5 \mathrm{~h}$ after microinjection. As shown in Fig. 2, the proportion of NT embryos showing PCC was the highest at $3 \mathrm{~h}$ post-injection. At $3 \mathrm{~h}$ postinjection, the addition of caffeine significantly increased the proportion of NT embryos showing PCC as compared with NT embryos cultured without caffeine.

\section{Effect of caffeine on p34 $4^{\text {cdc2 }}$ kinase activity of porcine oocytes matured in vitro}

We examined the effect of caffeine on $\mathrm{p} 34^{\text {cdc2 }}$ kinase activity of the $44 \mathrm{~h}$-matured cultured oocytes and for $8 \mathrm{~h}$ further $(44+8 \mathrm{~h})$. p3 $4^{\text {cdc2 }}$ kinase activity in $44 \mathrm{~h}$-matured oocytes did not significantly change $8 \mathrm{~h}$ after culture, while the addition of caffeine significantly increased
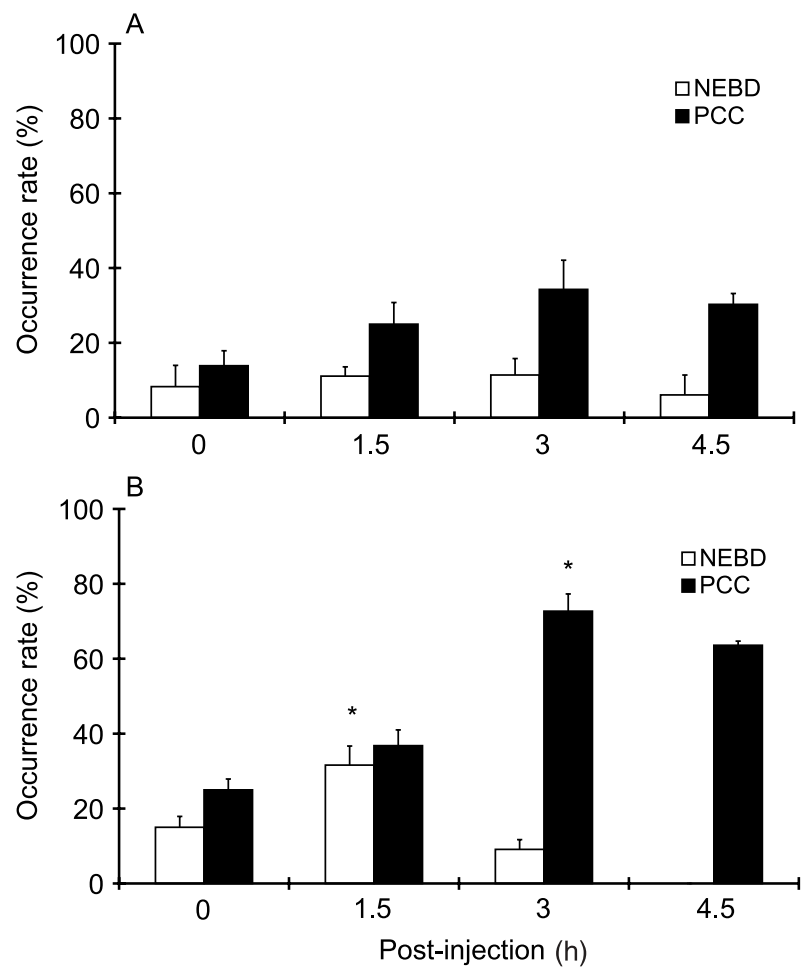

Figure 2 Effect of caffeine on chromosome configuration of porcine oocytes transferred with nuclei of fetal fibroblasts. Changes of chromosome configurations after nuclear transfer in treated without or with caffeine are shown in $\mathrm{A}$ or $\mathrm{B}$, respectively. The states of nuclei were examined after cultivation for $0,1.5,3$ and $4.5 \mathrm{~h}$. We categorized oocytes based on Fig. 1. NEBD: nuclear envelope breakdown, PCC: premature chromosome condensation, 1PN: one pronucleus, $2 \mathrm{PN}$ : two pronuclei. $*$ An asterisk in $\mathrm{B}$ denotes a significant difference $(P<0.05)$ from the bar at the same time point in $\mathrm{A}$. p3 $4^{\text {cdc2 }}$ kinase activity in comparison with both $44 \mathrm{~h}$ - and $44+8$ h-oocytes (Fig. 3A).

In addition, we examined how manipulation and supplementation of caffeine would affect p34 ${ }^{\text {cdc2 }}$ phosphorylation status in enucleated oocytes and NT embryos at $3 \mathrm{~h}$ after microinjection. As shown in Fig. 3B, enucleation did not affect p34 $4^{\text {cdc2 }}$ kinase activity of enucleated oocytes in the medium with or without caffeine. However, injection significantly decreased $\mathrm{p} 34^{\text {cdc2 }}$ kinase activity of NT embryos in the medium without caffeine. On the other hand, the addition of caffeine kept $\mathrm{p} 34^{\mathrm{cdc} 2}$ kinase activity of NT embryos to levels similar to those before microinjection.

\section{Effect of caffeine treatment during NT on in vitro development and abnormal pseudopronuclear formation of NT embryos}

The addition of caffeine during NT increased the rate of PCC in NT embryos. As shown in Table 1, the addition of caffeine significantly increased the blastocyst rate in comparison to other treatments. When NT embryos were activated at $0 \mathrm{~h}$ post-injection, no NT embryo developed to the blastocyst stage. Furthermore, the effect of the addition
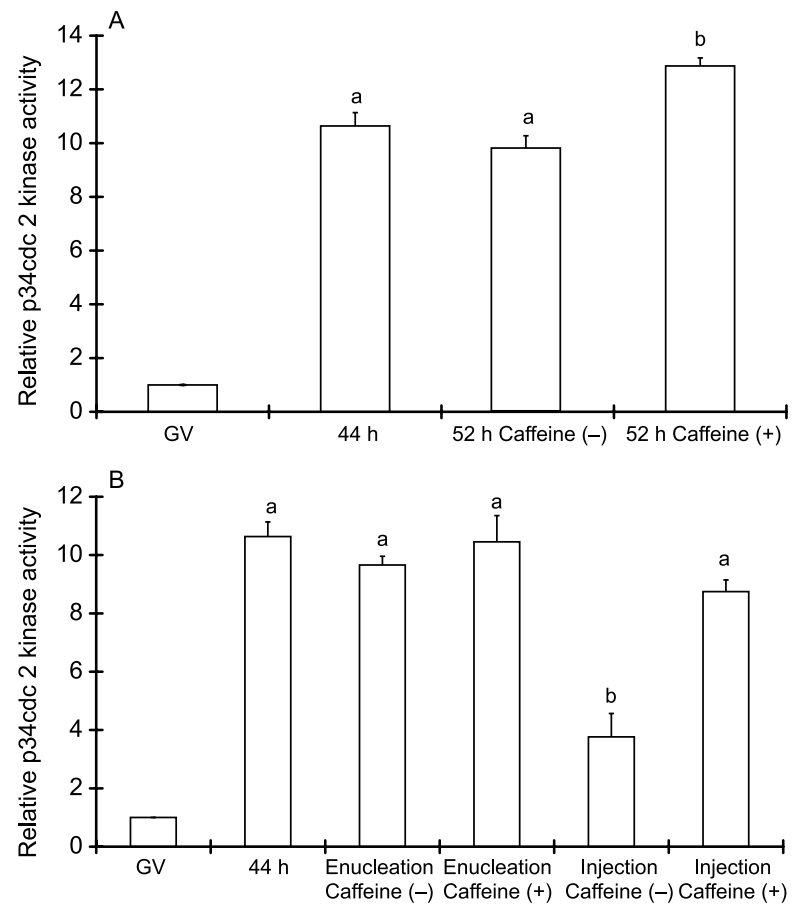

Figure 3 Effect of caffeine on $\mathrm{p} 34^{\mathrm{cdc} 2}$ kinase activity in porcine oocytes matured in vitro and during nuclear transfer (NT). Changes of p34 ${ }^{\text {cdc2 }}$ kinase activity in oocytes during maturation and NT are shown in A and B, respectively. (A) p34 $4^{\text {cdc2 }}$ kinase activity in the oocytes cultured for $8 \mathrm{~h}$ further in PB1 with or without $2.5 \mathrm{mM}$ caffeine after maturation. (B) p34 ${ }^{\text {cdc2 }}$ kinase activity in the oocytes enucleated and then injected in PB1 with or without $2.5 \mathrm{mM}$ caffeine. GV: germinal vesicle stage. ${ }^{\mathrm{a}-\mathrm{b}}$ Values with different superscripts are significantly different $(P<0.05)$. The level of the activity in GV oocytes is defined as 1 . 
Table 1 Effect of timing of activation and addition (+) of caffeine during NT on in vitro development of NT embryos.

\begin{tabular}{lcccc}
\hline $\begin{array}{l}\text { Addition of } \\
\text { caffeine }\end{array}$ & $\begin{array}{c}\text { No. of cultured } \\
\text { embryos }\end{array}$ & $\begin{array}{c}\text { No. (\%) of cleaved } \\
\text { embryos }\end{array}$ & $\begin{array}{c}\text { No. (\%) of embryos developed to } \\
\text { blastocyst stage (A) }\end{array}$ & $\begin{array}{c}\text { No. of cells in A } \\
\text { mean } \pm \text { s.E. }\end{array}$ \\
\hline IVF & 113 & $51(43.2)^{\mathrm{a}}$ & $37(32.9)^{\mathrm{a}}$ & $40.7 \pm 2.9^{\mathrm{a}}$ \\
$-^{*}$ & 103 & $31(30.1)^{\mathrm{a}}$ & $0(0.0)$ & $\mathrm{ND}$ \\
- & 122 & $68(55.7)^{\mathrm{a}}$ & $4(3.3)^{\mathrm{c}}$ & $45.0 \pm 8.1^{\mathrm{a}}$ \\
+ & 107 & $60(56.1)^{\mathrm{a}}$ & $11(10.3)^{\mathrm{b}}$ & $40.5 \pm 6.3^{\mathrm{a}}$ \\
\hline
\end{tabular}

*NT embryos were activated at $0 \mathrm{~h}$ post-injection, while the others were activated at $3 \mathrm{~h}$ post-injection. ${ }^{\text {a-c }}$ Values with different superscripts within the same column are significantly different $(P<0.05)$. ND, not determined.

of caffeine during NT on the pseudopronuclear formation was examined (Table 2). Although NT embryos were not artificially activated, NT embryos cultured without caffeine formed pseudopronuclei at 3 and $4.5 \mathrm{~h}$ post-injection, respectively $2.9 \%$ and $33.3 \%$. Meanwhile, when NT embryos were manipulated in the medium with caffeine, no NT embryo with pseudopronuclear formation was observed at any time point.

\section{Discussion}

In the present study, we showed that the addition of caffeine into the medium throughout NT increased the proportion of NT embryos showing PCC and kept p34 $4^{\text {cdc2 }}$ kinase activity high in NT embryos after microinjection. Furthermore, the present study revealed that this higher level of p34 $4^{\text {cdc2 }}$ kinase activity affects the quality of NT embryos with the resultant increase of blastocyst rate.

\section{Relationship between morphological remodeling and development of NT embryos}

In the mouse and bovine, the morphological remodeling of the transferred interphase nucleus may play an important role in subsequent development of NT embryos (Wakayama et al. 1998, Kim et al. 2002, Shin et al. 2002), suggesting that methods for induction of the morphological remodeling in NT embryos may result in increasing developmental potential. In addition, these reports showed evidence about the timing of activation of NT embryos and comparison between activated and inactivated cytoplasts, although in NT under conditions using inactivated cytoplasts the effect of increasing the proportion of NT embryos with PCC on further development was not examined (Wakayama et al. 1998, Tani et al. 2001). The morphological remodeling divides into two

Table 2 Effect of addition of caffeine during NT on pseudopronuclear formation in NT embryos without activation.

\begin{tabular}{lccc}
\hline $\begin{array}{l}\text { Addition } \\
\text { of caffeine }\end{array}$ & $\begin{array}{c}\text { No. of } \\
\text { cultured } \\
\text { embryos }\end{array}$ & $\begin{array}{c}\text { No. (\%) of NT } \\
\text { embryos formed } \\
\text { pseudopronucleus } \\
\text { at } \mathbf{3} \text { h post-injection }\end{array}$ & $\begin{array}{c}\text { No. (\%) of NT } \\
\text { embryos formed } \\
\text { pseudopronucleus } \\
\text { at } \mathbf{4 . 5} \text { h post-injection }\end{array}$ \\
\hline- & 35 & $1(2.9)$ & $11(33.3)$ \\
+ & 33 & $0(0.0)$ & $0(0.0)$ \\
\hline
\end{tabular}

www.reproduction-online.org types, known as NEBD and PCC (Kim et al. 2002). These changes are induced in interphase karyoplasts exposed with M-phase cytoplasts as a result of action of MPF (Yong \& Yuqiang 1998). However, despite the importance of the morphological remodeling for subsequent development, little information relevant to this subject is available in NT embryos of pigs. The results in this study indicate that the support/increase of MPF activity in NT embryos with caffeine promotes the formation of PCC in NT embryos, which improves further development after activation. On the other hand, no NT embryos activated just after microinjection developed to the blastocyst stage, suggesting that in these embryos morphological remodeling of transferred nucleus had not been completed. In both groups with or without caffeine treatment, there was no significant difference in the cleavage rate at day 2 or the average number of the nuclei of blastocysts at day 7 , while in the group treated with caffeine the blastocyst rate significantly increased in comparison to other groups. These results might be due to the differences of developmental quality among them. Yin et al. (2003) reported that the developmental potential was higher in oocytes reconstructed in the delayed-activated condition than in the simultaneously activated condition (Yin et al. 2003). They failed to produce cloned pigs by simultaneous activation although nine cloned piglets were produced by the delayed-activated condition. The present study also demonstrates that the advance of morphological remodeling of transferred nuclei may affect subsequent development of porcine NT embryos.

\section{MPF activity in porcine NT embryos}

MPF activity in cytoplasts plays a key role(s) for development of NT embryos, since the length of the maturation period affects the ability of porcine cytoplasts to support the development of NT embryos (Cheong et al. 2000, Ikeda \& Takahashi 2001, Miyoshi et al. 2002). In this study, we found that high MPF activity elevated in cytoplasts improved the development of NT embryos.

During prolonged culture, MPF activity of matured oocytes gradually decreases, as does the ability for fertilization and development (Kikuchi et al. 1995). In addition, it is known that the cultivation of matured oocytes for a long period decreases their quality as a cytoplast for NT (Cheong et al. 2000). In this study, p34 $4^{\text {cdc2 }}$ kinase activity, 
which is equal to MPF activity (Shimada et al. 2001), in matured oocytes did not significantly decrease without caffeine treatment by the further $8 \mathrm{~h}$ culture, which is equivalent to the manipulation period from enucleation to activation in this study. Therefore, the handling time for $8 \mathrm{~h}$ itself does not affect MPF activity in porcine oocytes.

To the contrary, the activity in oocytes and NT embryos changed dramatically throughout NT. When we assayed the MPF activity in the enucleated oocytes, we found that it was unchanged from intact matured oocytes. This result is in agreement with the report of Goto et al. (2002). Enucleation may not severely decrease MPF activity. On the other hand, we found that microinjection decreased MPF activity in NT embryos. This effect of microinjection seems not to be a result of aging during handling mentioned as above, but rather the influx of $\mathrm{Ca}^{2+}$ ions (Machaty et al. 1996). The influx of $\mathrm{Ca}^{2+}$ ions involved in microinjection could be avoided by performing manipulation in the $\mathrm{Ca}^{2+}$ ion-free medium; however, maintenance of porcine oocytes in a nondegenerated state requires the presence of $\mathrm{Ca}^{2+}$ ions in the medium (Bae \& Channing 1985). Thus, the influx of $\mathrm{Ca}^{2+}$ ions is intrinsic to NT procedure by microinjection. The present study indicated that the addition of caffeine into the handling medium prevented this decrease and is useful for support/ increase of MPF activity, resulting in the complete inhibition of the abnormal pseudopronuclear formation in porcine NT embryos without activation (Table 2). The balance between active MPF and pre-MPF in oocytes determines MPF activity. Caffeine inhibits this shift and increases MPF activity. Moreover, the addition of $2.0 \mathrm{mM}$ caffeine to IVF medium did not restrict subsequent development of IVF embryos (Table 1). In this report, it is possible that the addition of caffeine may inhibit the shift of MPF after injection in NT embryos. This method is very simple and may help us to produce NT embryos in all manipulations of porcine oocytes in vitro.

\section{Reprogramming of transferred nucleus and morphological remodeling by MPF}

In the present study, we showed that the support/increase of MPF activity until NT embryos received stimuli for activation, produced a high proportion of NT embryos with PCC, and resulted in increasing developmental ability to blastocyst in porcine NT embryos. In the mouse, when cumulus cells are transferred into inactivated cytoplasts, a change in chromosome structure to the DNase accessible state, and the redistribution of the TATA box binding protein occurs (Kim et al. 2002). In this aspect, since direct interactions of chromosomes with cytoplasmic factor(s) in oocytes may occur after NEBD, NEBD rather than PCC seems to play an important role in reprogramming by allowing the exchange of protein in transferred interphase nuclei. Considering that NEBD is followed by PCC, PCC may serve as an indicator of a success in nuclear remodeling. In this study, PCC was induced very shortly after NEBD in porcine NT embryos.

MPF is believed to cause PCC of transferred nuclei in NT embryos. Chromosome condensation is correlated with histone $\mathrm{H} 1$ and $\mathrm{H} 3$ phosphorylation, and histone $\mathrm{H} 1$ is often used as a substrate for MPF in vitro (Kubelka et al. 2002). However, the overall significance of H1 phosphorylation is not clear. Recently, Teranishi et al. (2004) observed the replacement of somatic linker histones with $\mathrm{H} 1$ foo, a mouse oocyte-specific linker histone. H1foo is thought to play an important role in nuclear remodeling, and the mobility of $\mathrm{H} 1$ foo may occur when the nuclear envelope does not exist. In a report on bovine NT, Tani et al. (2003) showed that the potential of nuclear transferred embryos to develop to the blastocyst stage was not different when somatic cells at the $M$ phase were fused with oocytes activated with ionomycin and cycloheximide $1-5 \mathrm{~h}$ before $(12-22 \%)$, but was significantly decreased when oocytes were activated $6 \mathrm{~h}$ before $(1 \%)$. This report indicates that there is no possibility for MPF to directly regulate reprogramming of donor cell nuclei, but it might be important to induce NEBD and/or PCC of interphase nuclei and to exclude the nuclear envelope of donor nuclei by transferring to oocytes with high MPF activity for exposing donor nuclei to the factor(s) involved in reprogramming. In the meantime, this is no problem in the case of transferring nucleus at the M-phase, because they do not have their nuclear envelope. The factor(s) involved in reprogramming might have a character not able to pass through the nuclear envelope of donor nuclei.

In conclusion, our results indicate that in pigs the addition of caffeine into the medium during NT increases the proportion of NT embryos with PCC via support/increase of MPF activity in the cytoplasm, followed by the resultant increase in the rate of development to the blastocyst stage.

\section{Acknowledgements}

Supported by the Program for Promotion of Basic Research Activities for Innovative Biosciences.

\section{References}

Bae IH \& Channing CP 1985 Effect of calcium ion on the maturation of cumulus-enclosed pig follicular oocytes isolated from mediumsized graafian follicles. Biology of Reproduction 33 79-87.

Boquest AC, Grupen CG, Harrison SJ, Mcllfatrick SM, Ashman RJ, d'Apice AJ \& Nottle MB 2002 Production of cloned pigs from cultured fetal fibroblast cells. Biology of Reproduction $\mathbf{6 6}$ $1283-1287$.

Cheong HT, Ikeda K, Martinez Diaz MA, Katagiri S \& Takahashi Y 2000 Development of reconstituted pig embryos by nuclear transfer of cultured cumulus cells. Reproduction, Fertility and Development 12 15-20.

De Sousa PA, Dobrinsky JR, Zhu J, Archibald AL, Ainslie A, Bosma W, Bowering J, Bracken J, Ferrier PM, Fletcher J et al. 2002 Somatic cell nuclear transfer in the pig: control of pronuclear formation and integration with improved methods for activation 
and maintenance of pregnancy. Biology of Reproduction $\mathbf{6 6}$ 642-650.

Funahashi H, Asano A, Fujiwara T, Nagai T, Niwa K \& Fraser LR 2000 Both fertilization promoting peptide and adenosine stimulate capacitation but inhibit spontaneous acrosome loss in ejaculated boar spermatozoa in vitro. Molecular Reproduction and Development 55 117-124.

Goto S, Naito K, Ohashi S, Sugiura K, Naruoka H, Iwamori N \& Tojo H 2002 Effects of spindle removal on MPF and MAP kinase activities in porcine matured oocytes. Molecular Reproduction and Development 63 388-393.

Ikeda K \& Takahashi Y 2001 Effects of maturational age of porcine oocytes on the induction of activation and development in vitro following somatic cell nuclear transfer. Journal of Veterinary Medical Science 63 1003-1008.

Kang YK, Koo DB, Park JS, Choi YH, Kim HN, Chang WK, Lee KK \& Han YM 2001 Typical demethylation events in cloned pig embryos. Clues on species-specific differences in epigenetic reprogramming of a cloned donor genome. Journal of Biological Chemistry 276 39980-39984.

Kawahara M, Mori T, Tanaka H \& Shimizu H 2002 The suppression of fragmentation by stabilization of actin filament in porcine enucleated oocytes. Theriogenology 58 1081-1095.

Kikuchi K, Izaike Y, Noguchi J, Furukawa T, Daen FP, Naito K \& Toyoda Y 1995 Decrease of histone H1 kinase activity in relation to parthenogenetic activation of pig follicular oocytes matured and aged in vitro. Journal of Reproduction and Fertility 105 325-330.

Kikuchi K, Naito K, Noguchi J, Shimada A, Kaneko H, Yamashita M, Aoki F, Tojo H \& Toyoda Y 2000 Maturation/M-phase promoting factor: a regulator of aging in porcine oocytes. Biology of Reproduction $63715-722$.

Kikuchi K, Naito K, Noguchi J, Kaneko H \& Tojo H 2002 Maturation/M-phase promoting factor regulates aging of porcine oocytes matured in vitro. Cloning Stem Cells 4 211-222.

Kim JM, Ogura A, Nagata M \& Aoki F 2002 Analysis of the mechanism for chromatin remodeling in embryos reconstructed by somatic nuclear transfer. Biology of Reproduction 67 760-766.

Kubelka M, Anger M, Pavlok A, Kalous J, Schultz RM \& Motlik J 2002 Activation of pig and cattle oocytes by butyrolactone I: morphological and biochemical study. Zygote 10 47-57.

Machaty Z \& Prather RS 1998 Strategies for activating nuclear transfer oocytes. Reproduction, Fertility and Development 10 599-613.

Machaty Z, Funahashi H, Mayes MA, Day BN \& Prather RS 1996 Effects of injecting calcium chloride into in vitro-matured porcine oocytes. Biology of Reproduction 54 316-322.

Miyoshi K, Umezu M \& Sato E 1999 Effect of hyaluronic acid on the development of porcine 1-cell embryos produced by a conventional or new in vitro maturation/fertilization system. Theriogenology $51777-784$.

Miyoshi K, Rzucidlo SJ, Pratt SL \& Stice SL 2002 Utility of rapidly matured oocytes as recipients for production of cloned embryos from somatic cells in the pig. Biology of Reproduction 67 $540-545$.
Prather RS, Hawley RJ, Carter DB, Lai L \& Greenstein JL 2003 Transgenic swine for biomedicine and agriculture. Theriogenology $\mathbf{5 9}$ $115-123$.

Quinn P, Barros C \& Whittingham DG 1982 Preservation of hamster oocytes to assay the fertilizing capacity of human spermatozoa. Journal of Reproduction and Fertility 66 161-168.

Shimada M, Zeng WX \& Terada T 2001 Inhibition of phosphatidylinositol 3-kinase or mitogen-activated protein kinase kinase leads to suppression of p34(cdc2) kinase activity and meiotic progression beyond the meiosis I stage in porcine oocytes surrounded with cumulus cells. Biology of Reproduction 65 $442-448$.

Shin MR, Park SW, Shim H \& Kim NH 2002 Nuclear and microtubule reorganization in nuclear-transferred bovine embryos. Molecular Reproduction and Development 62 74-82.

Tani T, Kato Y \& Tsunoda Y 2001 Direct exposure of chromosomes to nonactivated ovum cytoplasm is effective for bovine somatic cell nucleus reprogramming. Biology of Reproduction $\mathbf{6 4}$ 324-330.

Tani T, Kato Y \& Tsunoda Y 2003 Reprogramming of bovine somatic cell nuclei is not directly regulated by maturation promoting factor or mitogen-activated protein kinase activity. Biology of Reproduction 69 1890-1894.

Teranishi T, Tanaka M, Kimoto S, Ono Y, Miyakoshi K, Kono T \& Yoshimura Y 2004 Rapid replacement of somatic linker histones with the oocyte-specific linker histone $\mathrm{H} 1$ foo in nuclear transfer. Developmental Biology 266 76-86.

Uhm SJ, Chung HM, Kim C, Shim H, Kim NH, Lee HT \& Chung KS 2000 In vitro development of porcine enucleated oocytes reconstructed by the transfer of porcine fetal fibroblasts and cumulus cells. Theriogenology 54 559-570.

Wakayama T, Perry AC, Zuccotti M, Johnson KR \& Yanagimachi $R$ 1998 Full-term development of mice from enucleated oocytes injected with cumulus cell nuclei. Nature 394 369-374.

Yin XJ, Tani T, Yonemura I, Kawakami M, Miyamoto K, Hasegawa R, Kato Y \& Tsunoda Y 2002 Production of cloned pigs from adult somatic cells by chemically assisted removal of maternal chromosomes. Biology of Reproduction $67442-446$.

Yin XJ, Cho SK, Park MR, Im YJ, Park JJ, Jong Sik B, Kwon DN, Jun SH, Kim NH \& Kim JH 2003 Nuclear remodelling and the developmental potential of nuclear transferred porcine oocytes under delayed-activated conditions. Zygote 11 167-174.

Yong Z \& Yuqiang L 1998 Nuclear-cytoplasmic interaction and development of goat embryos reconstructed by nuclear transplantation: production of goats by serially cloning embryos. Biology of Reproduction 58 266-269.

Received 25 January 2005

First decision 18 March 2005

Revised manuscript received 14 April 2005

Accepted 23 May 2005 